\title{
PROGRAMA DE PREVENÇÃO E TRATAMENTO DO TABAGISMO: UMA VIVÊNCIA ACADÊMICA DE ENFERMAGEM
}

Verônica Cordeiro Machado ${ }^{1}$, Marli Isolina Alerico ${ }^{2}$, Janaina Sena ${ }^{3}$

RESUMO: O presente artigo relata a experiência como acadêmica de enfermagem no mês de julho de 2006, em um Programa de Prevenção e Combate ao Tabagismo da Secretaria Municipal de Saúde no Centro Municipal de Saúde do Município de Redentora-RS. Destaca-se a importância como acadêmica, de fazer parte deste projeto, o qual foi criado com os objetivos de auxiliar, orientar, tratar, recuperar, promover a saúde e prevenir as doenças, abordando as conseqüências que o tabaco traz para a vida da pessoa fumante e das que convivem com a mesma. Os resultados demonstraram ser significativos, pois algumas pessoas já deixaram o tabaco, outras diminuíram a quantidade consumida por dia, mas o mais relevante foi constatar que todos continuam o tratamento com o grupo de fumantes e a equipe profissional.

PALAVRAS-CHAVE: Enfermagem; Tabagismo; Promoção da saúde; Prevenção de doenças

\section{PROGRAM OF SMOKING PREVENTION AND TREATMENT: A NURSING ACADEMIC EXPERIENCE IN THE HEALTH AREA}

\begin{abstract}
The present article reports the experience, as a nursing undergraduate, of taking part in a Program of Smoking Prevention and Combat of the Municipal department of Health in the Municipal Health Center of Redentora/Brazil during the month of July/2006. Therefore, I underscore the importance as an undergraduate, to take part in this project, which was created to assist, to guide, to treat, to recover, to promote health and to prevent illness, approaching the consequences that tobacco brings to smokers' lives and people around them. The results showed to be meaningful as some people gave up the habit, others diminished the daily consumed amount, but the most important was to evidence that all of them continue the treatment with the peer group and the health team.
\end{abstract}

KEYWORDS: Nursing; Smoking; Health promotion; Disease prevention.

\section{PROGRAMA DE LA PREVENCIÓN Y TRATAMIENTO DEL EL FUMAR: UNA EXPERIENCIA ACADÉMICA EN EL ÁREA DE LA SALUD}

RESUMEN: El actual artículo trae la experiencia de una académica de enfermería en el mes de julio de 2006, en un programa de prevención y combate al tabaquismo de la Secretaria Municipal de Salud en la Centro Municipal de Salud de la ciudad de Redentora. Se destaca la importancia académica de se hacer ser parte de este proyecto, que fue creado para asistir, para dirigir, para tratar, para recuperar, para promover la salud y para prevenir las enfermedades, abordandolas consecuencias que el tabaco trae para la vida del fumador y de la gente que convive con él. Los resultados son significativos, pues algunos dejaron de fumar, otras disminuieron la cantidad consumida por día, pero el más importante es que todos continúan el tratamiento con el grupo y el equipo profesional.

PALABRAS CLAVE: Enfermería; Tabaquismo; Promoción de la salud; Prevención del enfermidades.

${ }^{1}$ Acadêmica. Curso de Graduação em Enfermagem da Universidade Regional Integrada do Alto Uruguai e das Missões, Campus de Frederico Westphalen.

${ }^{2}$ Enfermeira da Secretaria Municipal de Saúde do Município de Redentora/RS.

${ }^{3}$ Enfermeira. Mestre. Professora do Curso de Graduação em Enfermagem da Universidade Regional Integrada do Alto Uruguai e das Missões, Campus de Frederico Westphalen. 


\section{INTRODUÇÃO}

Atualmente, no Brasil e no mundo, o tabaco vem sendo consumido por milhões de cidadãos, os quais acabam sendo vítimas de diversas doenças causadas pelo consumo desta substância, tornandose um importante problema de saúde pública devido aos malefícios para quem é usuário e para aqueles que o rodeiam ${ }^{(1)}$.

O tabaco é uma planta cujo nome científico é Nicotina tabacum, da qual é extraída uma substância chamada nicotina. Seu uso surgiu aproximadamente no ano de 1.000 a.C., nas sociedades indígenas da América Central, em rituais mágicos-religiosos com o objetivo de purificar, contemplar, proteger e fortalecer os ímpetos guerreiros, além de acreditar que a mesma tinha o poder de predizer o futuro. A planta chegou ao Brasil provavelmente pela migração de tribos tupi-guaranis, sendo utilizada com fins curativos. No século seguinte, surgiu o hábito de aspirar rapé, ao qual foram atribuídas qualidades medicinais, pois a rainha da França, Catarina de Médicis, o utilizava para aliviar suas enxaquecas. Entre 1840 a 1850, surgiram as primeiras descrições de homens e mulheres fumando cigarros, porém somente após a Primeira Guerra Mundial (1914 a 1918) seu consumo apresentou grande expansão $0^{(2)}$.

Entende-se que o tabaco é um dos grandes causadores de problemas relacionados à saúde da população, pois é produzido e vendido em todo o mundo, constituindo não só desafios para os serviços de saúde, mas também para o desenvolvimento econômico, social, educacional e ambiental.

O tabagismo é amplamente reconhecido como uma doença resultante da dependência de nicotina, que leva os usuários dos produtos de tabaco a se exporem continuamente a uma ampla gama de doenças. Nessas doenças, estão relacionadas, entre outras, o câncer de boca, faringe e laringe, câncer de esôfago, câncer de pulmão, câncer de pâncreas, câncer de bexiga, cardiopatia isquêmica, hipertensão arterial, degeneração miocárdica, doença cardiopulmonar, aneurisma da aorta, doença vascular periférica, aterosclerose, bronquite crônica e enfisemas, tuberculose pulmonar, asma, pneumonia, úlcera péptica e outras doenças respiratórias ${ }^{(3)}$.

Existem ainda, outros agravos causados em parte pelo tabagismo, como o câncer de lábio, nariz, estômago, câncer de pelve do rim e do corpo do rim, peritonite, osteoporose, degeneração muscular relacionada ao envelhecimento e leucemia mielóide. O tabagismo acarreta, também, redução da fecundidade e traz malefícios para o desenvolvimento do feto, resultando em tamanho reduzido do recémnascido e a doença de Crohn. O tabagismo ainda pode prejudicar terceiros, como os que convivem com aquelas que fumam diariamente, filhos e pessoas que trabalham juntas ${ }^{(3)}$.

Pode-se dizer que o tabaco causa grandes malefícios à vida das pessoas, desencadeando diversos problemas, como doenças crônicas degenerativas, as quais causam, em muitos casos, sofrimento e dor. Devido à toxicidade do tabaco, o total de mortes decorrentes de seu uso é atualmente cerca de 5 milhões ao ano e, se tais tendências de expansão forem mantidas, as mortes alcançarão 8,4 milhões/ano em 2020. No Brasil, são estimadas cerca de $200 \mathrm{mil}$ mortes/ano em conseqüência do tabagismo ${ }^{(3)}$.

Frente aos dados alarmantes apresentados, entende-se que o tabaco, pelo seu alto índice de consumo em todo o mundo, se constitui em um sério problema de saúde pública que deve ser observado pelos órgãos públicos e responsáveis.

Diante do exposto, tendo como foco a preocupante situação do Brasil e, em particular do Município de Redentora, Rio Grande do Sul, a Secretaria Municipal de Saúde resolveu promover e recuperar a saúde de fumantes, incentivando o abandono dessa prática, bem como a diminuição do consumo a fim de prevenir e combater inúmeras doenças que podem vir a acometer pessoas que consomem tabaco.

Este trabalho tem como objetivo relatar a experiência vivenciada como acadêmica do sexto semestre de um curso de Graduação em Enfermagem durante a realização de estágio extra-curricular, no mês de Julho de 2006, em um Programa de Prevenção e Tratamento do Tabagismo.

\section{DESCRIÇÃO DO PROCESSO}

Após a observação de dados do mundo e do Brasil a respeito do elevado consumo de tabaco e suas conseqüências, a Secretaria Municipal de Saúde do município de Redentora, localizado no Noroeste Colonial do Rio Grande do Sul, implementou em suas ações de saúde o Programa de Prevenção e Tratamento do Tabagismo. Este programa foi criado com o intuito de promover e recuperar a saúde; prevenir as doenças; incentivar o não fumar e a diminuição do consumo de 
tabaco, como também orientar quanto às conseqüências e malefícios que o mesmo traz para a saúde do fumante e de quem convive com ele.

Segundo dados da Secretaria Municipal de Saúde, o município apresenta um índice elevado de fumantes e, devido a isso foi organizado e implementado este programa, o qual está sob coordenação de um enfermeiro, conta com o apoio de médico, farmacêutico e psicólogo, dois agentes comunitários de saúde e uma acadêmica de enfermagem. Para a implementação deste Programa foi elaborado um Plano, aprovado pela $15^{\mathrm{a}}$ Coordenadoria Regional de Saúde, e após o que foi encaminhado à Secretaria Estadual de Saúde e Ministério da Saúde, tendo sido aprovado.

O plano foi apresentado ao Prefeito Municipal que demonstrou interesse pelo tema, incentivando a implantação do mesmo. Em seguida, reuniu-se a equipe da Secretária Municipal de Saúde para informações de como estava organizada a proposta e, após isso, foi apresentado também aos agentes comunitários de saúde. Como uma das primeiras iniciativas, a Secretaria Municipal de Saúde regulamentou a resolução n. 001/06, restringindo o uso de tabaco e derivados do mesmo, que somente poderiam ser consumidos em áreas sinalizadas, denominadas de "fumódromos", o que ajudou, possibilitando maior ênfase para o andamento do programa.

Após a confirmação da aprovação, a Secretaria Municipal de Saúde, através do enfermeiro responsável e dos agentes comunitários de saúde, passou a divulgar o programa tentando atingir o maior número de pessoas. Além disso, se fez divulgação por meio da rádio local e intermunicipais e pelo jornal, com o intuito de que as pessoas que fumantes fossem despertadas e/ ou motivadas a participar do grupo que seria formado pelo Programa.

Para atender aos que procuraram pelo programa, foi feita a formação de grupos conforme horários pré-estabelecidos pela Secretaria Municipal de Saúde. Os trabalhos iniciaram-se em 28 de junho de 2006, quando aconteceu o acolhimento dos participantes, a apresentação do Programa, os objetivos e as metas de sua criação.

Os grupos foram subdivididos em três subgrupos, tendo encontros semanais, durante o primeiro mês. Estes quatro encontros eram as sessões/ programas preconizadas pelo Ministério da Saúde. Já no segundo mês os encontros passaram a acontecer quinzenalmente, e a partir do terceiro mês eram mensais, em que se realizava a manutenção, orientação e reavaliação dos pacientes. Todos os encontros eram em datas definidas estabelecidas para as quartas-feiras, nos horários: 8:30 horas para o primeiro subgrupo; 10 horas para o segundo subgrupo e 13:30 horas para o terceiro subgrupo.

O trabalho em grupo é uma realidade no cotidiano do trabalho de equipes de saúde, sendo vivenciado em toda a vida profissional, seja na atenção direta aos clientes ou na relação com a própria equipe, porém, é necessário a busca de maior reflexão sobre esta temática, para podermos utilizar o grupo de forma consciente, reflexiva e efetiva ${ }^{(4)}$.

Os grupos podem assumir diversos formatos conforme suas finalidades, podendo ser de trabalho, de treinamento, terapêuticos, entre outros. É difícil dizer o que é e o que não é psicoterapia de grupo, principalmente porque os grupos não terapêuticos podem atingir resultados semelhantes aos dos grupos terapêuticos ${ }^{(5)}$. Ainda, os resultados terapêuticos podem permear a vida de qualquer grupo, conforme a natureza que os caracteriza ${ }^{(6)}$

Após o chamamento das pessoas para participarem do grupo, foi efetuada a primeira consulta de avaliação clínica, sendo que 41 estiveram presentes, tendo sido realizada avaliação médica e de Enfermagem. No segundo encontro participaram 34 pessoas, em que foi realizada entrevista individual com cada uma e discussão sobre o que é tabaco, o malefício que ele causa para a saúde de quem fuma e de quem é de seu convívio, a importância de participar do grupo e a vontade que cada um deve demonstrar para abandonar o vício.

Considera-se a importância do atendimento individualizado para posterior inserção ao grupo, e que ações educativas são realizadas nas unidades básicas de saúde, individual e coletivamente. Há, porém, um destaque maior para a educação em saúde visando o coletivo. As ações desenvolvidas com grupos tornamse muito ricas em trocas de experiências e coletivamente as pessoas têm mais força de reivindicar $^{(7)}$.

No terceiro encontro, foi discutido sobre como seria o tratamento, os medicamentos que poderiam ser fornecidos aos participantes, como deveriam ser utilizados, as conseqüências e as reações que poderiam causar, os benéficios da diminuição do consumo de tabaco, a importância de se manter firme na decisão de abandonar o vício. Neste encontro, dois participantes informaram que haviam abandonado o uso do tabaco 
sem a utilização de medicamentos, somente com orientações e com o apoio do grupo, e isso nos pareceu um incentivo aos demais participantes do grupo.

No quarto encontro foi distribuída a medicação para os pacientes que receberam prescrição médica e, destes, todos aceitaram usá-la; independentemente da opção, ou não, do uso da medicação, eles poderiam participar do grupo. Quanto à medicação, é realizada orientação sobre o uso e tratamento com avaliação da equipe de saúde. Foi realizado, ainda, um teste individual com cada participante, para ver em qual escala de dependência cada um se encontrava. Este foi um momento de intensa reflexão, pois a cada participante foi solicitado falar quantos cigarros fumava por dia, o que trocaria pelo cigarro, se não fumasse o que sentiria e assim por diante; foram questões respondidas uma a uma por todos, de maneira individualizada.

O referido teste é denominado de Teste de Fagerstrom, utilizado para avaliação dos participantes e classifica os pontos quanto aos itens citados anteriormente da seguinte forma: 0 a 2: muito baixo; 3 e 4: baixo ; 5: médio; 6 e 7: elevado e 8 a 10: muito elevado. Assim, o teste classificou os pacientes em distintos grupos e em três níveis de dependência: baixo, elevado e muito elevado ${ }^{(8)}$.

O enfermeiro responsável pelo grupo, juntamente com a equipe de apoio, relatou que ficaram surpresos com os resultados obtidos após aplicação e avaliação do teste: com baixo grau de dependência do tabaco foram 10 pessoas; com grau elevado $22 \mathrm{e}$ com grau muito elevado foram constatadas 08 pessoas. Destas, não houve pacientes de muito baixo grau e de médio grau de dependência pelo uso do tabaco.

Para os 10 pacientes classificados com baixo grau de dependência do tabaco, foi indicado o tratamento com goma de mascar de nicotina. Destes, 4 abandonaram o cigarro após 8 dias, 3 abandonaram o grupo e outros 3 continuaram no tratamento e abordagem, com expectativa e vontade de parar de fumar, pois relatavam o desejo de abandonar o tabaco e que o grupo estava sendo um grande incentivador e responsável pelos resultados obtidos até o momento.

Os 22 pacientes classificados como grau "elevado" tiveram, como prescrição inicial de tratamento, o adesivo de $14 \mathrm{mg}$, na seqüência o adesivo de $7 \mathrm{mg}$ e depois a goma de mascar de nicotina. Destes, 6 pacientes tiveram o tratamento interrompido pela equipe de saúde devido a efeitos adversos do tratamento, pois apresentavam muita irritabilidade local pelo uso do adesivo, queixas de insônia, mudanças no humor, cefaléia, ansiedades, angústias e muita vontade de fumar; 2 pacientes foram encaminhados ao psicólogo; 4 pararam de fumar e, outros 12 pacientes continuam o tratamento e participam do grupo e das atividades realizadas.

Ainda, para os 10 pacientes classificados com grau "muito elevado" de dependência do tabaco, foi usado o tratamento adesivo de $21 \mathrm{mg}$, após de $14 \mathrm{mg}$ e depois de $7 \mathrm{mg}$ e, também a goma de mascar com nicotina. Destes pacientes, 04 pararam de fumar definitivamente e 06 continuaram o tratamento. Estes pacientes, com grau "elevado" de dependência, relatam grande dificuldade em fazer o tratamento, tendo o tabaco como um centro dominante. No entanto, com o apoio do grupo, o uso do medicamento e o incentivo que estão tendo, está sendo possível realizar o tratamento. Eles relataram diversas queixas como cefaléia, ansiedade, tontura, tosse, formigamento das mãos, irritabilidade, alterações do sono e repouso, dentre outras manifestações clínicas, as quais variam muito de paciente para paciente.

Como se pode observar, o Programa de Prevenção e Combate do Tabagismo, implementado pela Secretaria Municipal de Saúde, veio a contribuir para a melhoria da saúde da população de Redentora, pois trouxe ações educativas em saúde como prevenção de doenças, proporcionando o tratamento para quem quisesse deixar de fumar, com orientações, conversas e encontros mensais, nos quais são abordados reiteradamente os prejuízos que o tabaco traz para a saúde de quem fuma.

Para a avaliação dos resultados obtidos pelo grupo a equipe baseou-se no nível de percepção dos pacientes, na mudança de comportamento, na aceitação das orientações e na decisão de parar de fumar e, em conseqüência disso, traçou novas estratégias de ação com base no número de pessoas que pararam ou diminuíram o consumo de tabaco.

É necessário pensar a educação e a saúde não mais como uma educação sanitarizada, localizada no interior do campo da saúde, ou ainda uma educação para a saúde, como se a saúde pudesse ser um estado que se atingisse depois de educado. Porquanto, é preciso recuperar a dimensão da educação e do processo saúde-doença e estabelecer as articulações entre esses dois campos e os movimentos sociais percebendo-os como práticas sociais articuladas ${ }^{(9)}$.

Por fim, pode-se constatar que os resultados obtidos foram significativos, pois algumas pessoas já 
deixaram o tabaco, outras diminuíram a quantidade consumida por dia, mas o mais importante é constatar que todas continuam o tratamento com o grupo e a equipe. Elas perceberam e percebem que o tabaco causa malefício para a sua própria saúde e a de quem convive com um fumante.

\section{CONSIDERAÇÕES FINAIS}

Após este relato de experiência vivenciado como acadêmica de enfermagem no Programa de Prevenção e Combate do Tabagismo, tendo a possibilidade de participar desde o início em sua implementação e dos momentos nos grupos, resultou em uma experiência que contribuirá significativamente para a formação profissional. Isso é devido ao aprendizado e trocas de experiências e informações com o grupo, com profissionais envolvidos e, em especial, com o enfermeiro que permitiu conhecer um pouco mais das possibilidades da prática da Enfermagem, pois compartilhava suas expectativas e conquistas. Também aprendi muito com os pacientes, pelas suas experiências de serem tabagistas, com o desejo e as dificuldades de deixarem de fumar.

A partir disto, pode-se contatar que os objetivos do Programa estão sendo alcançados, pois percebemos que há pessoas que estão se conscientizando de que fumar causa males à saúde. Vários integrantes que iniciaram no primeiro grupo permanecem participando dos encontros e, o Programa foi ampliado com mais um grupo com 40 pessoas. Isso mostra que o trabalho da equipe de profissionais da Secretaria Municipal de Saúde, o enfermeiro e a equipe que apoiou o grupo está sendo bem sucedido, conseguindo alcançar a proposta inicial de promoção de saúde, prevenção das doenças e combate ao tabagismo.

Finalmente, após avaliação e mudanças de estratégias de ação para alcançar novos resultados, a Secretaria Municipal da Saúde continua com o Programa de Prevenção e Tratamento do Tabagismo, seguindo o protocolo do Ministério da Saúde, porém aumentando a equipe multidisciplinar e organizando novos grupos com no máximo 12 pacientes, acreditando em um atendimento mais individualizado, humanizado e garantindo o direito à saúde para todos.

\section{REFERÊNCIAS}

1. Ministério da Saúde (BR). Instituto Nacional de Câncer. Tabagismo e Saúde nos Países em Desenvolvimento Documento organizado pela Comissão Européia em
Colaboração com a organização Mundial de Saúde e o Banco Mundial para a Mesa Redonda de Alto Nível sobre Controle do Tabagismo e Políticas de desenvolvimento, 2003.

2. Serrat SM. Drogas e álcool: prevenção tratamento. Campinas: São Paulo Komedi; 2001.

3. Ministério de Saúde (BR). Portaria GM/MS/ N. ${ }^{\circ} 1.575$, de 29 de agosto de 2002. Consolida o Programa Nacional de Controle de Tabagismo, e dá outras providências. Diário Oficial da União, Brasília, DF, 3 Set. 2002.

4. Munardi DB, Rodrigues ARF. Enfermagem e grupos. Goiânia: AB; 1997.

5. Wood JK. Pequenos grupos centrados na pessoa: mais que terapia. Campinas: PCSG, 1990 (mimeografado).

6. Munardi DB, Zago MMF. Grupos de apoio/suporte e grupos de auto-ajuda: aspectos conceituais e operacionais, semelhanças e diferenças. Rev Enferm UERJ. 1997;5(1):359-66.

7. Rosso CFW, Collet N. Os enfermeiros e a prática de educação em saúde em municípios do interior paranaense. Rev Eletron Enferm. 1999;1(1).

8. Santos RD. Diretrizes Brasileiras Sobre Dislipidemias e Diretrizes de Prevenção da Aterosclerose do Departamento de Aterosclerose da Sociedade Brasileira de Cardiologia. Arquivos Bras Cardiol. 2001;77: (supl3).

9. Melo JAC. Educação sanitária: uma visão crítica. Cad Cedes Educ Saúde. 1987;4:28-43.

Cogitare Enferm. 2007 Abr/Jun; 12(2):248-52 